\title{
Temperature Effects on Electrode Processes. VII. The Effects of Solvent and Electrolyte on the Relative Entropies of Formation of Doubly and Singly Charged Ions of Organic Compounds
}

\author{
MORTEN SVAAN and VERNON D. PARKER
}

Laboratory for Organic Chemistry, Norwegian Institute of Technology, University of Trondheim, $\mathrm{N}$-7034 Trondheim-NTH, Norway

The ratios of the entropies of formation of doubly charged ions to those of the corresponding ion radicals, $\Delta S_{2} / \Delta S_{1}$, were observed to vary from 2.9 to 7.1 depending upon the nature of the substrate and the aprotic solvent. The data are compared to expected ratios of solvation energies estimated from the simple Born equation and found to correspond remarkably well. A comparison is also made with ratios of solvation energies of doubly and singly charged inorganic ions having the same inert gas electronic configuration. The latter are somewhat larger than the Born equation prediction. It is concluded that the studies of the entropies of formation of the doubly charged ions support the view that the entropies give a measure of the energetics of the solvation of the ions and hence very valuable information concerning the details of the electron transfer processes.

In the previous papers of this series we have reported the results of our investigations on the effect of substrate structure,${ }^{1-4}$ supporting electrolyte ${ }^{5}$ and solvent ${ }^{6}$ on the entropies of formation of ion radicals of aromatic compounds. We have concluded that the entropies are related to the free energies of formation of the ion radicals since the enthalpies can be considered to be constant over the temperature intervals in which the measurements by phase selective second harmonic a.c. voltammetry (SHAC) are made. To now, the entropies of formation have only been obtained for mono charged ions. In this paper we report the results of measurements on a

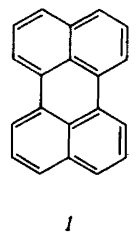

number of doubly charged ions in a number of different aprotic solvents.

According to the unmodified Born equation (1), where $N$ is Avogadros number, $e$ is the electronic charge, $D$ is the solvent dielectric constant and $r$ is the ionic radius, $\Delta G_{\text {solv }}$ is

$-\Delta G_{\text {solv }}=N e^{2}\left(1-D^{-1}\right) / 2 r$

predicted to be proportional to the square of the ionic charge. The solvent is treated as a dielectric continuum and the ions as spheres. This model then gives a prediction that $\Delta G_{\text {solv }}$ of either dianions or dications of aromatic compounds should be about 4 times the corresponding values for the mono charged ion of the same substrate. This follows from the fact that little difference is expected in the shape and structure of the aromatic ions. These considerations lead to the prediction that the entropies of formation for the doubly charged ions should be of the order of $4 x$ those of the corresponding ion radicals, providing that the steric effects toward solvation do not change appreciably in going from one to the other of the charged species.

It was the purpose of this work to obtain the entropies of formation of a number of doubly 
charged ions and compare these values with the corresponding ones for the formation of the ion radicals. The scope of the study is necessarily limited by the fact that not many aromatic dianions and dications are sufficiently stable to allow the reversible potentials to be measured to the degree of needed precision. Even in cases where the doubly charged ions are sufficiently stable the electrode processes are frequently complicated by adsorption phenomena. ${ }^{7,8}$ The results reported here are all for well defined electrode processes which fulfil the requirements for reversibility when measured by SHAC., ${ }^{9,10}$

\section{RESULTS AND DISCUSSION}

The data in Table 1 are for the consecutive $1 \mathrm{e}^{-}$ reductions of substrates to anion radical and to dianion in the same solvents used previously, ${ }^{6}$ acetonitrile $(\mathrm{MeCN}), N, N$-dimethylformamide (DMF), propylene carbonate (PC) and pyridine (PYR). The substrates used were the alternate aromatic hydrocarbon perylene (1), dibenzoylbenzene (2) and fluorenylidenemalonontrile (3).<smiles>O=C(C(=O)c1ccc(C(=O)c2ccccc2)cc1)c1ccccc1</smiles>

1<smiles>[O-]C(c1ccccc1)=c1ccc(=C([O-])c2ccccc2)cc1</smiles><smiles>C=[Tl]</smiles><smiles>[O-]C(c1ccccc1)=c1ccc(=C([O-])c2ccccc2)cc1</smiles><smiles>[O-]C(c1ccccc1)=c1ccc(=C([O-])c2ccccc2)cc1</smiles>

Scheme 1. 1,4-Dibenzoylbenzene.

Table 1. A comparison of the entropies of formation of anion radicals and dianions of aromatic compounds in aprotic solvents. ${ }^{a}$

\begin{tabular}{|c|c|c|c|c|}
\hline Substrate & Solvent & Charge change & $-\Delta S_{273.2}$ & $\Delta S_{2} / \Delta S_{1}$ \\
\hline Perylene & $\mathrm{MeCN}$ & $\begin{array}{r}0 \text { to }-1 \\
-1 \text { to }-2\end{array}$ & $\begin{array}{l}2.33 \\
12.6\end{array}$ & 6.40 \\
\hline Perylene & DMF & $\begin{array}{r}0 \text { to }-1 \\
-1 \text { to }-2\end{array}$ & $\begin{array}{l}3.48 \\
15.6\end{array}$ & 5.48 \\
\hline Perylene & PYR & $\begin{array}{r}0 \text { to }-1 \\
-1 \text { to }-2\end{array}$ & $\begin{array}{l}7.58 \\
18.4\end{array}$ & 3.43 \\
\hline 1,4-Dibenzoylbenzene & $\mathrm{MeCN}$ & $\begin{array}{r}0 \text { to }-1 \\
-1 \text { to }-2\end{array}$ & $\begin{array}{c}8.69 \\
42.7\end{array}$ & 5.91 \\
\hline 1,4-Dibenzoylbenzene & DMF & $\begin{array}{r}0 \text { to }-1 \\
-1 \text { to }-2\end{array}$ & $\begin{array}{l}9.32 \\
27.8\end{array}$ & 3.98 \\
\hline 1,4-Dibenzoylbenzene & PC & $\begin{array}{r}0 \text { to }-1 \\
-1 \text { to }-2\end{array}$ & $\begin{array}{l}9.62 \\
36.3\end{array}$ & 4.78 \\
\hline 1,4-Dibenzoylbenzene & PYR & $\begin{array}{r}0 \text { to }-1 \\
-1 \text { to }-2\end{array}$ & $\begin{array}{l}12.0 \\
20.0\end{array}$ & 2.67 \\
\hline $\begin{array}{l}\text { Fluorenylidene- } \\
\text { malononitrile }\end{array}$ & DMF & $\begin{array}{r}0 \text { to }-1 \\
-1 \text { to }-2\end{array}$ & $\begin{array}{l}2.40 \\
13.7\end{array}$ & 6.71 \\
\hline $\begin{array}{c}\text { Fluorenylidene- } \\
\text { malononitrile }\end{array}$ & $\mathrm{PC}$ & $\begin{array}{r}0 \text { to }-1 \\
-1 \text { to }-2\end{array}$ & $\begin{array}{l}2.22 \\
13.3\end{array}$ & 7.07 \\
\hline
\end{tabular}

${ }^{a}$ Solvent symbols defined in the text. 


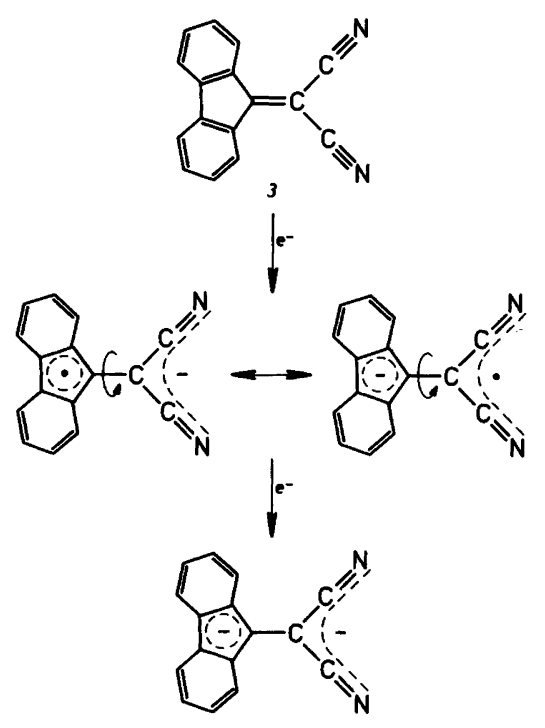

Scheme 2. Fluorenylidenemalononitrile.

The electrode processes for the latter two are illustrated in Schemes 1 and 2, respectively.

Since the entropy of formation of the dianion from the neutral substrate, abbreviated as $\Delta S_{2}$, is the quantity with which comparisons are to be made it is necessary to convert the entropy change observed in the electrode process (anion radical $\rightarrow$ dianion) to $\Delta S_{2}$ according to eqn. (2).

$$
\Delta S_{2}=\Delta S_{1}+\Delta\left(S_{2}-S_{1}\right)
$$

Having done this, the ratio $\Delta S_{2} / \Delta S_{1}$ can be calculated. The last column in Table 1 gives the values for the three substrates in the various solvents. Data are given in all cases when it was possible to make the measurements of the re-

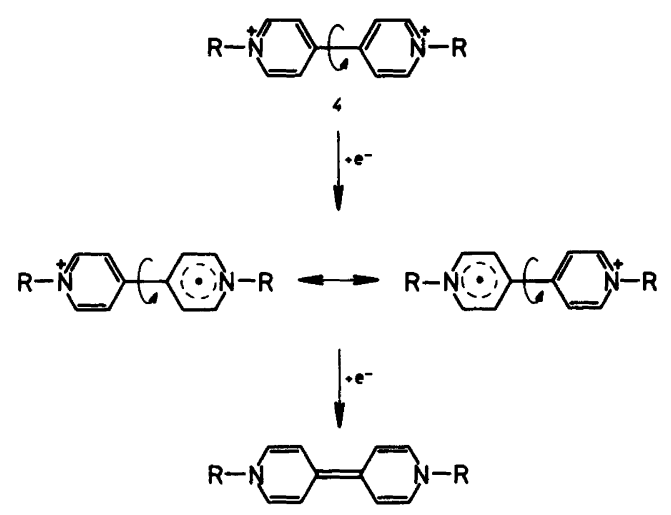

Scheme 3. Dialkyl viologens.

versible potentials by SHAC. The observed ratios vary from a low value of 2.7 to a high one of 7.1. The mean value was found to be 5.2 with a standard deviation of 1.5 . Thus, the ratios are slightly larger than that predicted for the solvation energies by the simple Born equation (1).

The data in Table 2 are for the reduction of the dialkylviologen dications (4) where $R$ is either methyl or heptyl. These stable dications are reduced first to the cation radical and then to the neutral species (Scheme 3). The ratios of the entropies of formation, $\Delta S_{2} / \Delta S_{1}$, are somewhat lower than those in Table 1 and also a bit lower than predicted by the Born equation. It is interesting to note that there is a substantial effect of the nature of $R$ on the entropies of formation of the ions with the heptyl substituted dication and cation radical having values of the order of twice those for the case where $R$ is methyl.

Since in some cases, the entropies of formation of cation and anion radicals have been observed

Table 2. A comparison of the entropy changes for the reductions of di- and mono-positive bipyridine salts in propylene carbonate. ${ }^{a}$

\begin{tabular}{lllll}
\hline$R^{b}$ & $X^{c}$ & Charge change & $\Delta S_{273.2} /(\mathrm{cal} / \mathrm{K} \mathrm{mol})$ & $\Delta S_{2} / \Delta S_{1}$ \\
\hline Methyl & $\mathrm{Cl}$ & +2 to +1 & 38.2 & 3.62 \\
& & +1 to 0 & 14.6 & \\
Heptyl & $\mathrm{Br}$ & +2 to +1 & 19.3 & 3.83 \\
& & +1 to 0 & 6.82 & \\
\hline
\end{tabular}

\footnotetext{
${ }^{a}$ Measurements at a gold electrode by phase selective second harmonic a.c. voltammetry in solvent containing $\mathrm{Bu}_{4} \mathrm{NBF}_{4}(0.10 \mathrm{M})$ over a temperature range of $50 \mathrm{~K}{ }^{b}$ The substituent in structure $4 .^{c}$ The halide in structure 4.
} 
Table 3. The effect of electrolyte concentration on the entropies of formation of the cation radicals and dications of 5,10-dimethyl-5,10-dihydrophenazine in acetonitrile. ${ }^{a}$

\begin{tabular}{llll}
\hline$\left[\mathrm{Bu}_{4} \mathrm{NBF}_{4}\right] / \mathrm{m}$ & Charge change & $\Delta S_{273.2} /(\mathrm{cal} / \mathrm{K} \mathrm{mol})$ & $\Delta S_{2} / \Delta S_{1}$ \\
\hline 0.033 & 0 to +1 & 10.6 & 3.30 \\
& +1 to +2 & 24.4 & \\
0.067 & 0 to +1 & 11.6 & 3.34 \\
& +1 to +2 & 27.2 & \\
0.130 & 0 to +1 & 13.1 & 2.92 \\
& +1 to +2 & 25.1 & \\
\hline
\end{tabular}

${ }^{a}$ As in Table 2.

to be strongly affected by the supporting electrolyte concentration in acetonitrile, a series of experiments were carried out on the successive oxidations of 5,10-dimethyl-5,10-dihydrophenazine (5) to the cation radical and dication (Scheme 4). With $\left[\mathrm{Bu}_{4} \mathrm{NBF}_{4}\right]$ ranging from 0.033 to $0.13 \mathrm{M}$, very little effect was found on the entropies and hence $\Delta S_{2} / \Delta S_{1}$ (Table 3). This result was not anticipated since the medium effect on the formation of the dication was expected to be amplified due to the higher charge. However, since the observed values are very nearly constant and not too far removed from the value predicted by the Born equation

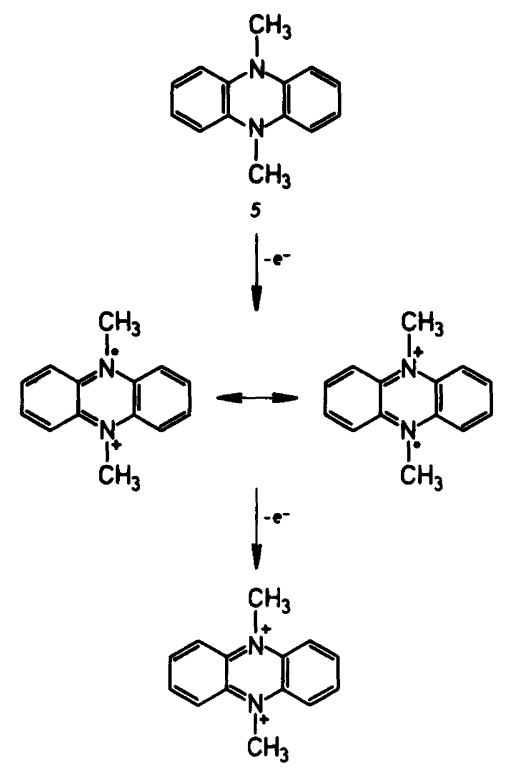

Scheme 4. 5,10-Dimethyl-5,10-dihydrophenazine. this result supports those previously discussed and strengthens the indication that $\Delta S_{2} / \Delta S_{1}$ is not very sensitive to changes in the medium.

It is of interest to compare literature data ${ }^{11}$ for the free energies of solvation of spherical inorganic ions with the entropies reported here. The data selected from Ref. 11 allow ions of roughly the same size to be compared as ratios of doubly to singly charged. The data reported ${ }^{11}$ for water and $\mathrm{MeCN}$ as solvents along with the corresponding electron configurations of the ions are shown in the upper part of Table 4 . Then making the assumption that ions with the same inert gas core configuration will be of roughly the same size, the ratios of the solvation energies of doubly charged to singly sharged ions were calculated. The most interesting feature of the results, listed in the lower part of Table 4 , is that the ratios were found to be almost independent of the identity of the ions and to be only slightly larger in $\mathrm{MeCN}$. The mean of all the values was found to be 7.20 with a standard deviation of 1.3.

The ratios found for the inorganic ions are somewhat larger than those found for the various types of aromatic substrates. These are also considerably greater than predicted by the simple Born eqn. (1). The Born equation is known to be inadequate and various modifications have been discussed. ${ }^{12}$ So the deviations of parameters for either the inorganic ions or those studied here are not unexpected. The fact that the entropy ratios for the aromatic ions are considerably lower than the solvation energy ratios for the inorganic ions suggests that other factors must be considered for the aromatic ions. It has been the overall conclusion of all of the studies in this series ${ }^{1-6}$ that steric effects of solvation play an important role in the entropies of formation. It is conceiv- 
Table 4. A comparison of the solvation energies of mono and doubly charged inorganic ions with the same inert gas core electron configuration. ${ }^{a}$

\begin{tabular}{|c|c|c|c|c|}
\hline Ion & Electron configuration & $\begin{array}{l}-\Delta G_{\text {solv }}^{\circ} / \mathrm{e} . \mathrm{V} \\
\mathrm{H}_{2} \mathrm{O}\end{array}$ & $\mathrm{MeCN}$ & \\
\hline $\begin{array}{l}\mathrm{Cl}^{-} \\
\mathrm{Br}^{-} \\
\mathrm{Zn}^{2+} \\
\mathrm{Cd}^{2+} \\
\mathrm{Cu}^{2+} \\
\mathrm{Ca}^{2+}\end{array}$ & $\begin{array}{l}\text { argon core } \\
\text { krypton core } \\
\text { argon core }+3 \mathrm{~d}^{10} \\
\text { krypton core }+4 \mathrm{~d}^{10} \\
\text { argon core }+3 \mathrm{~d}^{9} \\
\text { argon core }\end{array}$ & $\begin{array}{r}70.7 \\
64.9 \\
486.6 \\
430.5 \\
497.2 \\
380.8\end{array}$ & $\begin{array}{r}55.7 \\
54.2 \\
477.5 \\
427.6 \\
519.9 \\
368.8\end{array}$ & \\
\hline $\operatorname{Ion}^{2+} /$ ion $^{-}$ & & \multicolumn{2}{|l|}{$\Delta G_{\mathrm{solv}}^{\mathrm{o} 2+} / \Delta G_{\mathrm{solv}}^{\mathrm{o}-}$} & Average $^{b}$ \\
\hline $\begin{array}{l}\mathrm{Zn}^{2+} / \mathrm{Cl}^{-} \\
\mathrm{Cu}^{2+} / \mathrm{Cl}^{-} \\
\mathrm{Ca}^{2+} / \mathrm{Cl}^{-} \\
\mathrm{Cd}^{2+} / \mathrm{Br}^{-} \\
\text {mean }\end{array}$ & & $\begin{array}{l}6.88 \\
7.03 \\
5.39 \\
6.09\end{array}$ & $\begin{array}{l}8.57 \\
9.33 \\
6.62 \\
7.68\end{array}$ & $\begin{array}{l}7.73 \\
8.18 \\
6.01 \\
6.89 \\
7.20\end{array}$ \\
\hline
\end{tabular}

${ }^{a}$ Solvation energy data from Ref. $11 .{ }^{b}$ The average value for the two solvents.

able that these do not differ appreciably for mono and doubly charged ions and that this is an additive factor which has the effect of reducing the ratio of the entropies of formation of the different ions. We are not suggesting that $\Delta S_{2} /$ $\Delta S_{1}$ should fall in the same range as the ratios in Table 4, if it were possible to strip the values of the contribution from steric effects. On the other hand if it were possible to separate the effects, a closer correspondence would probably be found.

Our general conclusion is that the studies of the entropies of formation of the doubly charged ions support our view that the entropies give a measure of the energetics of the solvation of the ions and hence very valuable information concerning the details of the electron transfer processes.

\section{EXPERIMENTAL}

The measurement procedure was the same as the one described previously in some detail. ${ }^{1}$ Solvent and electrolyte purification remained as in earlier papers and all substances were recrystallized before use.

\section{REFERENCES}

1. Svaan, M. and Parker, V. D. Acta Chem. Scand. B 35 (1981) 559.

2. Svaan, M. and Parker, V. D. Acta Chem. Scand. B 36 (1982) 351.

Acta Chem. Scand. B 38 (1984) No. 9
3. Svaan, M. and Parker, V. D. Acta Chem. Scand. B 36 (1982) 357.

4. Svaan, M. and Parker, V. D. Acta Chem. Scand. B 36 (1982) 365.

5. Svaan, M. and Parker, V. D. Acta Chem. Scand. B 38 (1984) 751.

6. Svaan, M. and Parker, V. D. Acta Chem. Scand. B 38 (1984) 759.

7. Hammerich, O. and Parker, V. D. J. Am. Chem. Soc. 96 (1974) 4284.

8. Jensen, B. S. and Parker, V. D. J. Chem. Soc. Chem. Commun. (1974) 367.

9. McCord, T. G. and Smith, D. E. Anal. Chem. 41 (1969) 1423.

10. Ahlberg, E. and Parker, V. D. Acta Chem. Scand. B 34 (1980) 91.

11. Case, B. and Parsons, R. Trans. Faraday Soc. 63 (1967) 1224.

12. Criss, C. M. and Salomon, M. In Covington, A. K. and Dickenson, T., Eds., Physical Chemistry of Organic Solvent Systems, Plenum, London and New York 1973, Chapter 2.

Received January 4, 1984. 